http://dx.doi.org/10.1590/1678-4162-8017

Arq. Bras. Med. Vet. Zootec., v.67, n.6, p.1653-1659, 2015

\title{
Efeito da inclusão da torta de macaúba na população de protozoários do rúmen de vacas leiteiras
}

\author{
[The effect of macauba meal inclusion in the ruminal protozoa \\ population of dairy cows] \\ A.C.R. dos Santos ${ }^{1}$, D.Q. Magalhães ${ }^{1}$, R.A. Azevedo ${ }^{2}$, I.L.N.L. Vieira ${ }^{1}$, D.E.G. França ${ }^{1}$, \\ L.C. Geraseev ${ }^{1 *}$, E.R. Duarte ${ }^{1}$ \\ ${ }^{1}$ Instituto de Ciências Agrárias - UFMG - Montes Claros, MG \\ ${ }^{2}$ Escola de Medicina Veterinária - UFMG - Belo Horizonte, MG
}

\begin{abstract}
RESUMO
Avaliaram-se os efeitos da inclusão da torta de macaúba (TM) na dieta sobre a concentração e o perfil da população de protozoários ciliados do rúmen. Foram utilizadas oito vacas Holandesas, dispostas em dois quadrados latinos 4x4 e alimentadas com diferentes níveis de inclusão da TM $\left(0,100,200\right.$ e $300 \mathrm{~g} \mathrm{~kg}^{-1} \mathrm{de}$ matéria seca na dieta total). Para quantificação e identificação dos protozoários, amostras de $1 \mathrm{~mL}$ do líquido ruminal de cada animal foram diluídas em $9 \mathrm{~mL}$ de solução de formaldeído a $10 \%$. Após diluições decimais sucessivas em solução salina, os protozoários foram quantificados em câmara de Sedgewick. A identificação foi realizada sob a luz da microscopia óptica, utilizando-se a objetiva de 40x para caracterizar o mínimo de 100 indivíduos por amostra. As concentrações de protozoários pequenos $(\mathrm{P}<0,01)$, médios $(\mathrm{P}=0,05)$, grandes $(\mathrm{P}=0,01)$ e o número total $(\mathrm{P}<0,01)$ apresentaram comportamento linear decrescente com o aumento dos níveis de inclusão da TM nas dietas. Foram encontradas características morfológicas típicas de 16 gêneros de protozoários ciliados, sendo os gêneros Entodinium e Eodinium os mais frequentes no líquido ruminal dos animais avaliados. O perfil da população de protozoários ciliados sofreu pequenas alterações com a inclusão da TM, sendo observada alteração na participação percentual dos gêneros Charonina $(\mathrm{P}=0,03)$ e Polyplastron (P $<0,01)$. A utilização da TM na dieta de bovinos promove redução na concentração e modificação no perfil da população de protozoários ciliados do rúmen.
\end{abstract}

Palavras-chave: pecuária leiteira, ciliados do rúmen, Acrocomia aculeada, coproduto

\begin{abstract}
The effects of meal macaúba (MM) inclusion on the concentration and profile of the ciliate protozoa of the bovine rumen were evaluated. Eight Holstein cows were used during second lactation, with average $480 \mathrm{~kg}$ of body weight, arranged in two $4 \times 4$ Latin squares simultaneously and fed four diets with different concentrations of the MM $\left(0,100,200\right.$ and $\left.300 \mathrm{~g} \mathrm{~kg}^{-1}\right)$. For identification and quantification of protozoa, samples of ruminal fluid from each animal with $1 \mathrm{ml}$ were diluted in $9 \mathrm{~mL}$ of $10 \%$ formaldehyde. After consecutive decimal dilutions in saline solution, protozoa were assessed in a Sedgewick chamber. The identification was carried out under the optical light microscope using a 40x objective to characterize at least 100 individuals per sample. The concentrations of small $(P<0.01)$, medium $(P=0.05)$, large $(P=0.01)$, and the total number $(P<0.01)$ of protozoa decreased linearly with the increasing MM inclusion. Morphological characteristics typical of 16 genera of ciliates were found, being the Entodinium spp. and Eodinium spp. the most frequent in the rumen fluid of animals evaluated. The profile of the population of ciliated protozoa suffer minor changes with the addition of $M M$, with a change in the percentage share of Charonina spp. $(P=0.03)$ and Polyplastron spp. $(P$ $<0.01)$. The use of the $M M$ in the cattle diet promotes reduction in the concentration and change in the profile of the population of rumen ciliates protozoa.
\end{abstract}

Keywords: dairy livestock, rumen ciliates, Acrocomia aculeate, byproduct

Recebido em 24 de setembro de 2014

Aceito em 19 de junho de 2015

*Autor para correspondência (corresponding author)

E-mail: lgeraseev@gmail.com 


\section{INTRODUÇÃO}

A macaúba (Acrocomia aculeata (Jacq.) Lodd. Ex Mart.) é uma espécie nativa das florestas tropicais e se destaca por ser uma palmeira oleaginosa altamente produtiva e adaptada a regiões semiáridas (Braga, 1976). O fruto da macaúba tem despertado grande interesse socioeconômico por se tratar de uma importante fonte de óleo vegetal, podendo ser utilizado para a produção de biodiesel, de cosméticos e de alimentos (Coimbra e Jorge, 2012). Durante o processo de extração do óleo por prensagem hidráulica, são geradas grandes quantidades de biomassa residual, podendo representar mais de $500 \mathrm{~g} \mathrm{~kg}^{-1}$ do total do fruto processado.

Essa biomassa residual é denominada torta de macaúba (TM), a qual apresenta altas concentrações de carboidratos fibrosos e de lipídeos, sendo indicada como alternativa econômica na alimentação de ruminantes (Azevedo et al., 2012; Azevedo et al., 2013a), podendo ou não provocar alterações nos padrões de consumo (Azevedo et al., 2013b; Azevedo et al., 2013c).

Os protozoários ocorrem em número de, aproximadamente, $10^{6}$ células por mililitro de conteúdo ruminal e são, na maioria, ciliados. Os ciliados dividem-se em dois grandes grupos: os holotrichas, que possuem cílios por todo o corpo, e os entodiniomorfos, que somente possuem cílios em regiões discretas, geralmente adjacentes ao citóstoma. Os holotrichas podem engolfar grânulos de amido, os quais são degradados mais lentamente que a degradação efetuada por bactérias ruminais, o que pode contribuir para evitar queda brusca do $\mathrm{pH}$ em animais alimentados com dietas ricas em concentrado. Já os entodiniomorfos são capazes de aderirem-se às fibras e possuem atividade celulolítica e hemicelulolítica (Williams e Coleman, 1985).

Os protozoários ciliados do rúmen têm crescimento estimulado ou inibido por fatores dietéticos (Williams, 1986). A suplementação lipídica em excesso na dieta de ruminantes pode comprometer o crescimento microbiano ruminal (Palmquist e Jenkins, 1980; Byers e Schelling, 1989), sendo as bactérias Gram-positivas, metanogênicas e os protozoários os mais sensíveis (Doreau e Ferlay, 1995).

Dado o teor elevado de lipídeos na torta de macaúba, objetivou-se com esta pesquisa avaliar o efeito do fornecimento do coproduto sobre a concentração e o perfil da população de protozoários ciliados do rúmen de vacas leiteiras.

\section{MATERIAL E MÉTODOS}

Os procedimentos adotados com os animais foram aprovados pelo Comitê de Ética em Experimentação Animal da Universidade Federal de Minas Gerais (Protocolo: 87/2008). Foram utilizadas oito vacas Holandesas com $480 \pm 26 \mathrm{~kg}$ de peso corporal, com média de produção de $21,4 \pm 3,8 \mathrm{~kg}$ por dia, na segunda lactação, idade média de 4,1 \pm 0 ,2anos e com 45 dias de lactação. Os animais foram alojados em baias cobertas e individuais, com seis metros quadrados, piso de terra batida, bebedouros automáticos, comedouros e saleiros individuais. As unidades experimentais foram distribuídas em dois quadrados latinos $4 \times 4$, balanceados para efeito residual, sendo quatro diferentes níveis de inclusão da TM $\left(0,100,200\right.$ e $\left.300 \mathrm{~g} \mathrm{~kg}^{-1}\right)$ na dieta total e quatro períodos experimentais. Os períodos experimentais foram de 21 dias cada, sendo os primeiros 14 dias de adaptação dos animais à dieta e os últimos sete dias para realização das coletas de dados.

As dietas experimentais foram formuladas de acordo com as recomendações do NRC (2001) para produção estimada de $25 \mathrm{~kg}$ de leite, com níveis crescentes de inclusão da TM na matéria seca (MS; Tab. 1). O volumoso e o concentrado foram misturados nos cochos e fornecidos aos animais duas vezes ao dia, em duas porções iguais, uma pela manhã (sete horas) e outra à tarde (17h), ajustados de forma a manter as sobras em $200 \mathrm{~g} \mathrm{~kg}^{-1}$ do oferecido. As amostras dos alimentos fornecidos foram coletadas diariamente, sendo identificadas e acondicionadas em freezer para posteriores análises laboratoriais. Todas as amostras foram pré-secas em estufa ventilada a $55^{\circ} \mathrm{C}$ e, posteriormente, moídas em moinho com peneira de um milímetro. Logo após, foram acondicionadas em frascos identificados, para posterior análise. 
Tabela 1. Ingredientes e composição química das dietas $\left(\mathrm{g} \mathrm{kg}^{-1}\right.$ de matéria seca) e da torta de macaúba (TM)

\begin{tabular}{lccccc} 
& \multicolumn{3}{c}{ Concentrações $\left(\mathrm{g} \mathrm{kg}^{-1}\right)$ da TM na dieta } & TM \\
\cline { 2 - 4 } & 0 & 100 & 200 & 300 & \\
\hline Ingredientes & & & & & \\
Silagem de milho & 500,0 & 500,0 & 500,0 & 500,0 & - \\
Caroço de algodão & 100,0 & 100,0 & 100,0 & 100,0 & - \\
Milho moído & 339,8 & 242,4 & 143,0 & 53,5 & - \\
Farelo de soja & 74,3 & 72,2 & 72,6 & 64,3 & - \\
Torta de macaúba & 0,0 & 100,0 & 200,0 & 300,0 & - \\
Ureia + SA (1\%) & 6,7 & 8,5 & 10,1 & 13,0 & - \\
Calcário calcítico & 6,0 & 5,0 & 3,9 & 2,8 & - \\
Fosfato bicálcico & 2,1 & 2,8 & 3,6 & 4,4 & - \\
Premix mineral-vitamínico & 4,5 & 4,5 & 4,5 & 4,5 & - \\
Composição química & & & & & \\
Matéria seca & 565,0 & 563,4 & 563,0 & 568,8 & 878,7 \\
Cinza & 139,7 & 119,4 & 118,2 & 117,1 & 50,1 \\
Proteína bruta & 147,6 & 147,6 & 147,8 & 147,9 & 66,4 \\
Extrato de etéreo & 47,8 & 64,0 & 80,0 & 95,8 & 197,3 \\
Fibra em detergente neutro & 430,9 & 479,8 & 538,2 & 547,3 & 720,3 \\
Carboidratos não fibrosos & 234,0 & 189,2 & 115,8 & 91,9 & - \\
\hline
\end{tabular}

${ }^{1}$ Composição de ácidos graxos da TM (por kg de ácidos graxos): 3,2g C12:0; 2,6g C14:0; 205,2g C16:0; 39,5g C16:1 cis-9; 15,0g C18:0; 490,9g C18:1 cis-9; 51,1g C18:1 cis-11, 14,3g C18:1 cis-12; 5,8g C18:1 cis-13; 154,0g C18:2 cis9 cis-12; 8,8 g C18:3 n-3; 5,9g outros ácidos graxos; 228,0g ácidos graxos saturados; 768,3g ácidos graxos insaturados; 602,9g ácidos graxos monoinsaturados; 165,4g ácidos graxos poli-insaturados.

As amostras foram analisadas de acordo com a Association of Official Analytical Chemists (AOAC, 1997) para MS (método 934,01), cinzas (método 942,05), proteína bruta (PB, método 954,01) e extrato etéreo (EE, método 920,39). Fibra em detergente neutro (FDNa, Van Soest et al., 1991) foi analisada no ANKOM200 Fiber Analyzer (ANKOM Technology Corporation, Fairport, Nova York, EUA). Sulfito de sódio e alfa-amilase foram usados na determinação de FDNa. O teor de carboidratos não fibrosos foi calculado como: $\mathrm{CFN}=100-(\% \mathrm{FDNa}+\% \mathrm{EE}$ $+\%$ PB $+\%$ de cinzas), de acordo com o NRC (2001).

A composição de ácidos graxos da TM foi determinada de acordo com Machmu Èller e Kreuzer (1999). Realizou-se extração com clorofórmio-metanol $(2: 1, \mathrm{v} / \mathrm{v})$ e metilação dos lipídeos, sendo posteriormente determinados e quantificados os ácidos graxos em cromatógrafo de gás HP 5890A (Hewlett-Packard, Avondale, Pensilvânia, EUA), equipado com um Supelcowax 10-coluna (coluna capilar de sílica fundida, $30 \mathrm{~m} \times 0,32 \mathrm{~mm})$.
Ao final de cada período experimental, os animais foram submetidos a jejum de sólidos por 12 horas, sendo, em seguida, imobilizados em brete de contenção para coletar amostra do líquido ruminal. Após a contenção, foi realizada a tricotomia e a assepsia, com solução de polivinilpirrolidona-iodo diluído a $1 \%$, em uma área de aproximadamente cinco centímetros quadrados, localizada na parte ventral do abdômen esquerdo, abaixo da fossa paralombar e cranialmente à articulação do joelho (Dirksen, 1993). Puncionou-se aproximadamente $15 \mathrm{~mL}$ de líquido ruminal, com o auxílio de cateter (SolidorR, 14.2, Bio Med Health Care Products, Haryana, Índia) acoplado a uma seringa estéril. As amostras foram armazenadas em tubos de ensaio e transportadas em caixas isotérmicas, a $4^{\circ} \mathrm{C}$, para o laboratório.

Os tubos de ensaio foram homogeneizados em vórtex, durante um minuto, e alíquotas de $1000 \mu \mathrm{L}$ foram transferidas para tubos contendo $9,0 \mathrm{~mL}$ de formaldeído a 10\%, para a conservação dos protozoários. Posteriormente, foram realizadas diluições decimais sucessivas em solução salina, para a determinação do 
número de protozoários em câmaras de Sedgewick (S52 glass, Pyser-SGI, Edenbridge, Kent, Reino Unido). As concentrações de protozoários pequenos (até $40 \times 60 \mu \mathrm{m}$ ), médios (até $100 \times 150 \mu \mathrm{m}$ ) e grandes (maior que $100 \mathrm{x}$ $150 \mu \mathrm{m})$ por mililitros de líquido ruminal foram determinadas após a visualização em microscópio óptico, utilizando-se a objetiva de 10x (Dirksen, 1993).

A identificação dos protozoários foi realizada em lâminas e lamínulas, com adição de uma gota da amostra em solução de $10^{-3}$ e uma gota de solução de lugol. A leitura e a identificação foram realizadas sob a luz da microscopia óptica, utilizando-se a objetiva de 40x para caracterizar o mínimo de 100 indivíduos por amostra. Os gêneros de protozoários foram classificados segundo as características morfológicas descritas por Dehority (1993).

Os resultados foram analisados de acordo com o procedimento GLM do SAS (versão 9.0; SAS Institute Inc., Cary, Carolina do Norte, EUA), em dois quadrados latinos $4 \times 4$, para testar o efeito da inclusão da TM sobre a concentração e o perfil da população de protozoários ciliados do rúmen. Os dados de concentração de protozoários foram analisados após transformação logarítmica $\left(\log ^{10}\right)$, e, em seguida, análise de regressão foi realizada para todas as variáveis obtidas no estudo. Valores de probabilidade inferiores ou iguais a 0,05 foram considerados significativos.

\section{RESULTADOS E DISCUSSÃO}

As concentrações de protozoários pequenos $(\mathrm{P}<0,01)$, médios $(\mathrm{P}=0,05)$ e grandes $(\mathrm{P}=0,01)$ e $\mathrm{o}$ número total de protozoários $(\mathrm{P}<0,01)$ decresceram linearmente com o aumento dos níveis de inclusão da TM nas dietas (Tab. 2). Os menores números de protozoários ciliados no líquido ruminal de animais alimentados com TM podem estar associados à menor ingestão de CNF e ao maior consumo de EE (Azevedo et al., 2014), uma vez que a carência de carboidratos solúveis resulta em diminuição significativa na quantidade de protozoários e a suplementação lipídica em excesso causa efeitos deletérios sobre o crescimento microbiano ruminal (Palmquist e Jenkins, 1980; Byers e Schelling, 1989).

Tabela 2. Concentrações $\left(\times 10^{4} / \mathrm{mL}\right.$ de líquido ruminal) de protozoários ciliados no líquido ruminal de vacas leiteiras alimentadas com diferentes níveis de inclusão de torta de macaúba (TM)

\begin{tabular}{|c|c|c|c|c|c|c|c|}
\hline & \multicolumn{4}{|c|}{ Concentrações $\left(\mathrm{g} \mathrm{kg}^{-1}\right)$ da TM na dieta } & \multirow{2}{*}{ EPM } & \multicolumn{2}{|c|}{ Valor de P } \\
\hline & 0 & 100 & 200 & 300 & & Linear & Quadrático \\
\hline \multicolumn{8}{|c|}{ Protozoários $^{1}$} \\
\hline Pequeno & 20,92 & 11,31 & 4,59 & 3,09 & 1,350 & $<0,01$ & 0,75 \\
\hline Médio & 1,78 & 0,88 & 0,19 & 0,23 & 0,045 & 0,05 & 0,86 \\
\hline Grande & 1,17 & 0,40 & 0,09 & 0,07 & 0,035 & 0,01 & 0,79 \\
\hline Total & 23,87 & 12,59 & 4,87 & 3,39 & 1,750 & $<0,01$ & 0,67 \\
\hline
\end{tabular}

${ }^{1}$ Pequeno (até 40 x $60 \mu \mathrm{m}$ ), médio (até $100 \times 150 \mu \mathrm{m}$ ) e grande (maior do que $100 \times 150 \mu \mathrm{m}$ ).

Essa diminuição na concentração de protozoários pode estar associada, ainda, à elevada concentração de ácidos graxos insaturados e à concentração considerável de ácidos graxos láurico (C12:0) e mirístico (C14:0) na TM (Tab. 1). Nesse sentido, pesquisas realizadas in vitro e in vivo demonstraram que os protozoários são sensíveis aos ácidos graxos insaturados oleico (C18:1 cis-9), linoleico (C18:2 cis-9 cis-12) e $\alpha$ linolênico (C18:3 n-3) (Machmüller et al., 1998; Hristov et al., 2004) e aos ácidos graxos saturados láurico e mirístico (Dohme et al., 1999; Faciola et al., 2013), sendo os dois últimos os que apresentam maior efeito tóxico sobre os protozoários ciliados do rúmen (Machmüller et al., 1998).

Os protozoários grandes foram os que apresentaram maior sensibilidade à atividade antiprotozoária da TM. O consumo de 100, 200 e $300 \mathrm{~g} \mathrm{~kg}^{-1}$ da TM diminuiu a concentração de protozoários grandes em 66, 92, 92,4 e 95,9\% (isto é, em relação à dieta sem TM), respectivamente. Já as concentrações de protozoários pequenos e médios foram reduzidas, respectivamente, em 46, 78 e $50,6 \% ; 78,1$ e 
$89,4 \%$ e 85,23 e $87,1 \%$, quando comparadas às concentrações de protozoários de animais mantidos com a dieta sem TM. Esses resultados evidenciam que a TM, quando adicionados à dieta, proporciona um potente efeito antiprotozoário.

Foram encontrados gêneros de protozoários das subclasses Holotricha (Buetschilia, Isotricha e Dasytricha) e Entodiniomorpha (Charonina, Entodinium, Diplodinium, Eodinium, Eremoplastron, Eudiplodinium, Diploplastron, Polyplastron, Ostracodinium, Elytroplastron, Enoploplastron, Ophyroscolex e Epidinium), com predominância dos entodiniomorfos, que corresponderam em mais de $70 \%$ da fauna, nos quatro níveis testados
(Tab. 3). Nesse contexto, observou-se que, nos animais mantidos com a dieta sem TM, houve predominância dos gêneros Eodinium e Entodinium, que corresponderam a 33,8 e a $33,3 \%$ dos protozoários ciliados identificados, respectivamente. Já nos animais mantidos com as dietas contendo $100 \mathrm{e}$ $200 \mathrm{gkg}^{-1}$ do coproduto, o gênero de maior predominância foi o Entodinium, correspondendo, respectivamente, a 35,4 e a $30,0 \%$ dos protozoários identificados. Com a inclusão de $300 \mathrm{~g} \mathrm{~kg}^{-1}$ da TM, os gêneros mais frequentes passaram a ser novamente $\mathrm{o}$ Eodinium e o Entodinium, correspondendo, respectivamente, a 28,0 e a $25,4 \%$ dos ciliados encontrados.

Tabela 3. Participação percentual dos gêneros de protozoários ciliados no líquido ruminal de vacas leiteiras alimentadas com diferentes níveis de inclusão de torta de macaúba (TM)

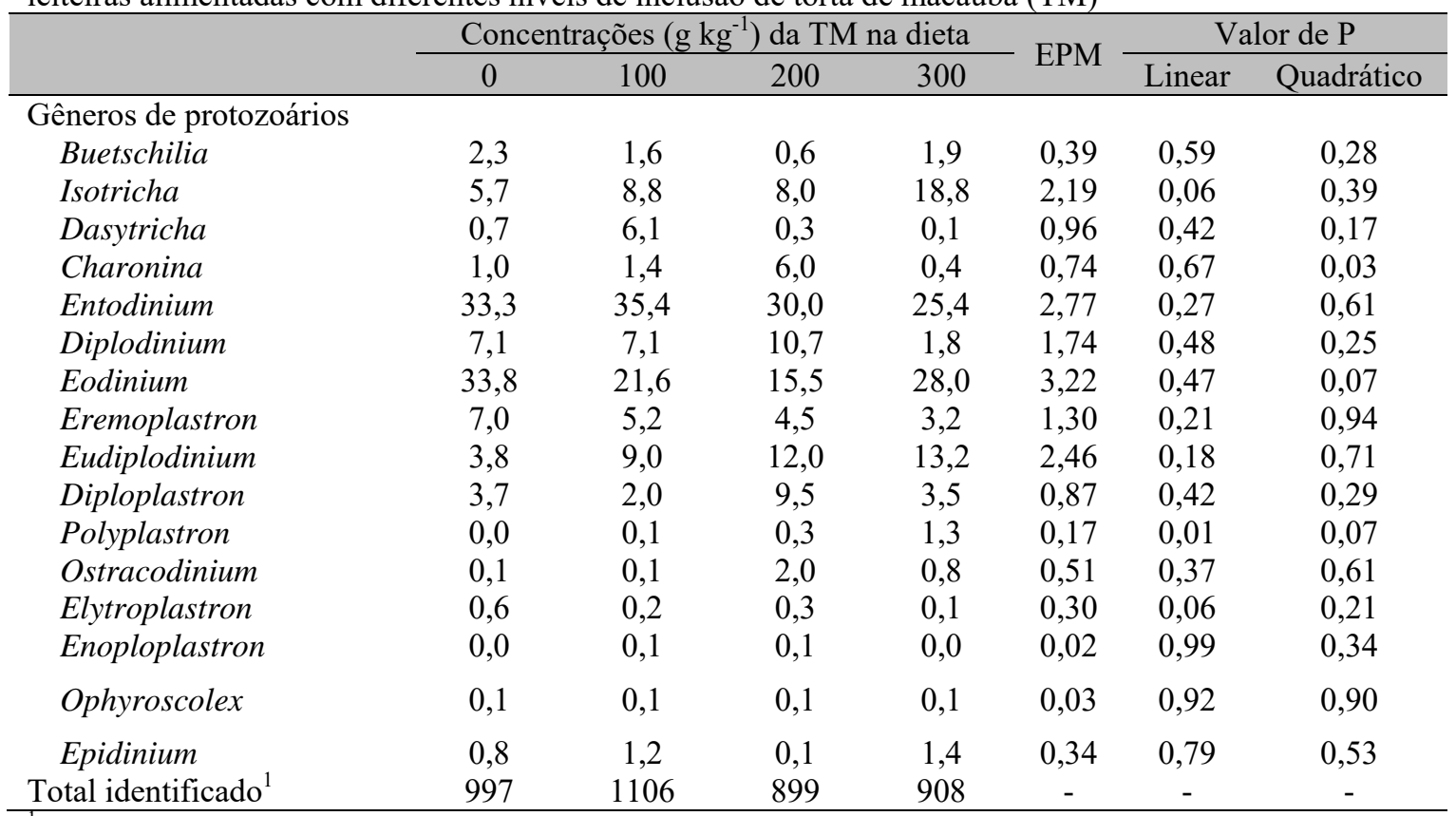

${ }^{\mathrm{I}}$ Número total de protozoários identificados por grupo de tratamento.

A predominância do gênero Entodinium está de acordo com as observações dos autores que investigaram as populações de protozoários no rúmen de bovinos alimentados com dietas contendo óleo de soja, associado ou não à monensina (Martinele et al., 2008), de ovinos criados na caatinga (Matos et al., 2008), de bovinos e bubalinos alimentados com dietas suplementadas com monensina ou própolis (Risponli et al., 2009) e em caprinos submetidos à dieta com casca de soja, em substituição ao milho (Carvalho et al., 2011). Essa predominância pode ser justificada por esse gênero apresentar várias espécies de ampla distribuição e ocorrência entre vários ruminantes domésticos e selvagens (Ogimoto e Imai, 1981).

A proporção do gênero Charonina apresentou comportamento quadrático $(\mathrm{P}=0,03)$ com $\mathrm{o}$ aumento da inclusão da TM nas dietas, o que pode estar associado ao mesmo comportamento quadrático da ingestão de FDN pelos animais 
(Azevedo et al., 2014), uma vez que ambos apresentaram valores máximos em $200 \mathrm{~g} \mathrm{~kg}^{-1}$ do coproduto na dieta. Essa observação é similar à de Rufino et al. (2011), os quais, ao avaliarem o líquido ruminal de caprinos alimentados com diferentes níveis de inclusão da TM, em substituição ao caroço de algodão, verificaram a predominância do gênero Charonina no líquido ruminal de animais alimentados com dieta contendo $100 \mathrm{~g} \mathrm{~kg}^{-1}$ da TM na matéria seca total.

Aumentos nos níveis de inclusão da TM resultaram em aumento linear $(\mathrm{P}<0,01)$ na participação percentual do Polyplastron spp., o que pode estar associado também ao consumo de FDN (Azevedo et al., 2014), uma vez que os entodiniomorfos são capazes de aderir-se às fibras, engolfam material particulado e possuem enzimas que digerem a celulose e a hemicelulose (Williams e Coleman, 1985). Dessa forma, Charonina spp. e Polyplastron spp. poderiam ter utilizado os carboidratos fibrosos como fonte de energia para o crescimento. Esse aumento na participação percentual do gênero Polyplastron pode estar associado, ainda, à maior tolerância desse gênero aos efeitos antiprotozoários da TM.

A atividade antiprotozoária da TM na dieta poderia ter efeito positivo na eficiência de utilização da energia da dieta, uma vez que os protozoários podem ser responsáveis por até $37 \%$ de metanogênese (Finlay et al., 1994), e, portanto, uma redução dos ciliados pode resultar em menor perda de energia a partir do rúmen. Além disso, pesquisadores têm relatado que a supressão de protozoários está intimamente relacionada com a redução da concentração de amônia no rúmen e o aumento da eficácia da síntese de proteína microbiana (Faciola et al., 2013; Fiorentini et al., 2013).

Entre as implicações dos efeitos da adição da TM nas dietas desses animais, poderia ocorrer redução na produção de metano, aumento de proteína bacteriana disponível para o animal e melhora da eficiência de utilização da energia das dietas. Futuros estudos são importantes para avaliar a produção de metano entérico com adição de TM na dieta de vacas leiteiras para indicá-la como possível prebiótico.

\section{CONCLUSÕES}

A utilização da torta de macaúba na dieta de bovinos promove redução na concentração de protozoários ciliados no líquido ruminal. A inclusão de $300 \mathrm{~g} \mathrm{~kg}^{-1}$ da torta de macaúba na dieta quase proporciona uma defaunação, indicando que esse coproduto apresenta um potente efeito antiprotozoário. Entretanto, o perfil da população de ciliados é pouco alterado com a inclusão da torta de macaúba.

\section{AGRADECIMENTOS}

Ao Conselho Nacional de Desenvolvimento Científico e Tecnológico (CNPq), à Fundação de Amparo à Pesquisa de Minas Gerais (Fapemig), à Coordenação de Aperfeiçoamento de Pessoal de Nível Superior (Capes) e PRPq / UFMG.

\section{REFERÊNCIAS}

AZEVEDO, R.A.; BICALHO, F.L.; ARAUJO, L. et al. Análise técnico-econômica de diferentes níveis da torta da torta de macaúba em dietas para vacas leiteiras. Arch. Zootec., v.62, p.1-4, $2013 \mathrm{a}$.

AZEVEDO, R.A.; RUFINO, L.M.A.; DOS SANTOS A.C.R. et al. Desempenho de cordeiros alimentados com inclusão de torta de macaúba na dieta. Pesqui. Agropecu. Bras., v.47, p.1663-1668, 2012.

AZEVEDO, R.A.; RUFINO, L.M.A.; DOS SANTOS, A.C.R. et al. Comportamento ingestivo de cordeiros alimentados com torta de macaúba. Arq. Bras. Med. Vet. Zootec., v.65, p.490-496, 2013b.

AZEVEDO, R.A.; SANTOS A.C.R.; RIBEIRO JÚNIOR, C.S. et al. Comportamento ingestivo de vacas alimentadas com torta de macaúba. Cienc. Rural, v.43, p.1485-1488, 2013c.

AZEVEDO, R.A.; SANTOS A.C.R.; RIBEIRO JÚNIOR, C.S. et al. Desempenho de vacas em lactação alimentadas com dietas contendo torta de macaúba. Arq. Bras. Med. Vet. Zootec., v.66, p.211218, 2014.

BRAGA, R. Plantas do nordeste, especialmente do Ceará. Fortaleza, Ceará: Escola Superior de Agricultura de Mossoró, 1976

BYERS, F.M.; SCHELLING G.T. Lipids in ruminant nutrition. In: CHURCH, D.C. (ed.). The ruminant animal: digestive physiology and nutrition. Englewood Cliffs, New Jersey: Prentice Hall, 1989. p.293-312. 
CARVALHO, L.F.P.; AMORIM, G.L.; MATOS, D.S. et al. Protozoários do rúmen de caprinos submetidos a dieta com casca de soja. Rev. Bras. Saúde Prod. Anim., v.12, p.244-253, 2011.

COIMBRA, M.C.; JORGE, N. Fatty acids and bioactive compounds of the pulps and kernels of Brazilian palm species, guariroba (Syagrus oleraces), jerivá (Syagrus romanzoffiana) and macaúba (Acrocomia aculeate). J. Sci. Food Agric., v.92, p.679684, 2012.

DEHORITY, B.A. Laboratory manual for classification and morphology of rumen ciliate protozoa. Boca Raton, Florida: CRC Press, 1993.

DIRKSEN, G. Sistema digestivo. In: DIRKSEN, G.; GRUNDER, H.D.; STOBER, M. (Eds.). Rosenberger: exame clínico dos bovines. Rio de Janeiro: Guanabara Koogan, 1993. p.166-169.

DOHME, F.; MACHMÜLLER, A.; ESTERMANN, B.L. et al. The role of the rumen ciliate protozoa for methane suppression caused by coconut oil. L. Appl. Microbiol., v.29, p.187-192, 1999.

DOREAU. M; FERLAY. A. Effect of dietary lipids on nitrogen metabolism in the lumen: a review. Livest. Prod. Sci., v.43, p.97-110, 1995.

FACIOLA, A.P.; BRODERICK, G.A.; HRISTOV, A. et al. Effects of lauric acid on ruminal protozoal numbers and fermentation pattern and milk production in lactating dairy cows. J. Anim. Sci., v.91, p.363-373, 2013.

FINLAY, B.J.; ESTEBAN, G.; CLARKE, K.J. et al. Some rumen ciliates have endosymbiotic methanogens. FEMS Microbiol. Lett., v.117, p.157$161,1994$.

FIORENTINI, G.; MESSANA, J.D.; DIAN, P.H.M. et al. Digestibility, fermentation and rumen microbiota of crossbred heifers fed diets with different soybean oil availabilities in the rumen. Anim Feed Sci. Technol., v.181, p.26-34, 2013.

HRISTOV, A.N.; IVAN, M.; MCALLISTER, T.A. In vitro effects of individual fatty acids on protozoal numbers and on fermentation products in ruminal fluid from cattle fed a high-concentrate, barley-based diet. J. Anim. Sci., v.82, p.2693-2704, 2004.

MACHMU ÈLLER, A.; KREUZER, M. Methane suppression by coconut oil and associated effects on nutrient and energy balance in sheep. Can. J. Anim. Sci.,v.79, p.65-72, 1999.
MACHMÜLLER, A.; OSSOWSKI, D.A.; WANNER, M. et al. Potential of various fatty feeds to reduce methane release from rumen fermentation in vitro (Rusitec). Anim. Food Sci. Technol., v.71, p.117-130, 1998.

MARTINELE, I.; EIFERT, E.C.; LANA, R.P. et al. Efeito da monensina e do óleo de soja sobre os protozoários ciliados do rúmen e correlação dos protozoários com parâmetros da fermentação ruminal e digestivos. Rev. Bras. Zootec., v.37, p.1129-1136, 2008.

MATOS, D.S.; GUIM, A.; BATISTA, A.M.V. et al. População de protozoários ciliados no rúmen de ovinos criados na caatinga de Pernambuco. Rev. Bras. Saúde Prod. Anim., v.9, p.270-279, 2008.

NUTRIENT requirements of dairy cattle. 7.ed. Washington: National Academy Press, 2001.

OFFICIAL methods of analysis. 16.ed. AOAC, Arlington, Virginia: AOAC, 1997.

OGIMOTO, K.; IMAI, S. Atlhas of rumen microbiology. Tokyo: Japan Scientific Societies Press, 1981.231p.

PALMQUIST, D.L.; JENKINS, T.C. Fat in lactation rations: review. J. Dairy Sci., v.63, p.1-14, 1980.

RISPOLI, T.B.; RODRIGUES, I.L.; MARTINS NETO, R.G. et al. Protozoários ciliados do rúmen de bovinos e bubalinos alimentados com dietas suplementadas com monensina ou própolis. Pesqui. Agropecu. Bras., v.44, p.92-97, 2009.

RUFINO, L.M.A.; BARRETO, S.M.P.; DUARTE, E.R. et al. Efeitos da inclusão de torta de macaúba sobre a população de protozoários ruminais de caprinos. Rev. Bras. Zootec. v.40, p.899-903, 2011.

STATISTICAL Analisys System for windows.Versão 9.1. Cary: SAS Inst. Inc., 2004.

VAN SOEST, P.J.; ROBERTSON, J.B.; LEWIS, B.A. Methods for dietary fiber, neutral detergent fiber, and nonstarch polysaccharides in relation to animal nutrition. J. Dairy Sci., v.74, p.3583-3597, 1991.

WILLIAMS AG. Rumen holotrich ciliate protozoa. Microbiol. Rev., v.50, p.25-49, 1986.

WILLIAMS, A.G.; COLEMAN, G.S. Hemicellulosedegrading enzymes in rumen ciliate protozoa. Curr. Microbiol., v.12, p.85-90, 1985. 